Z Gerontol Geriat 2011 · 44:83-84 DOI 10.1007/s00391-010-0161-2

Online publiziert: 12. April 2011

(c) Springer-Verlag 2011

\author{
C. Sieber ${ }^{1} \cdot$ W. v. Renteln-Kruse ${ }^{2}$ \\ ${ }^{1}$ Institut für Biomedizin des Alterns, Universität Erlangen-Nürnberg, \\ Medizinische Klinik 2, Klinikum Nürnberg \\ 2 Zentrum für Geriatrie und Gerontologie, Universität Hamburg, \\ Medizinisch-Geriatrische Klinik, Albertinen-Haus
}

\title{
Leitlinien in der geriatrischen Versorgung
}

eng geschnürtes Schema; dies ist letztendlich wohl auch der Hauptgrund, weshalb es gerade für diese speziell vulnerable Bevölkerungsgruppe nur wenige Leitlinien gibt. Eines der Ziele der Arbeitsgruppen der Deutschen Gesellschaft für Geriatrie (DGG) ist es deshalb, solche Leitlinien - wenn möglich mit den relevanten Fachgesellschaften zusammen - zu erarbeiten.

\section{$>$ Ziel muss es sein, Leitlinien für geriatrische Patienten zu erarbeiten}

In ihrem Beitrag zeigt $D$. Volkert auf, dass dies im Bereich der Ernährungstherapie gut gelungen ist. So konnten die Leitlinien der DGG, die zusammen mit der Deutschen Gesellschaft für Ernährungsmedizin (DGEM) 2004 erarbeitet wurden, später als Grundgerüst für die europäischen Leitlinien (2006) dienen. Momentan sind wir daran, die Leitlinien von 2004 wieder zu überarbeiten. Wichtig ist aber auch der Hinweis der Autorin, dass wenngleich Leitlinien bestehen, ihre Implementierung auf breiter Ebene im Bereich der Geriatrie nicht unproblematisch ist.

Die Kardiologie gilt als eine der medizinischen Disziplinen mit der breitesten Datengrundlage für die Entwicklung von Leitlinien. Der Beitrag von B. Frilling zeigt exemplarisch an der bevölkerungsmedizinisch bedeutsamen Erkrankung Herzinsuffizienz, dass auch hier die Datenlage für Empfehlungen zur Therapie älterer Patienten begrenzt ist. Tatsächlich evidenzbasierte Empfehlungen für ältere $\mathrm{Pa}$ tienten mit Herzinsuffizienz sind deshalb noch eher selten.
Anhand der Leitlinie für die nichtinvasive Beatmung illustrieren H.-J. Heppner et $a l$., dass sich die Intensivmedizin der Geriatrie gegenüber öffnet. Dies ist ein Beispiel, in dem sich eine Fachgesellschaft (Pneumologie und Beatmungsmedizin) an die DGG wandte, bei der Bearbeitung einer Leitlinie aktiv mitzuhelfen. Dies erfolgte sicher auch aus dem Gedanken heraus, dass die nichtinvasive Beatmung gerade bei den multimorbiden Betagten mit Frailty-Syndrom eine gute Option darstellt.

Die Beiträge beleuchten sowohl den Mangel als auch die Herausforderungen bei der Erstellung von Leitlinien für geriatrische Patienten. Die Bedeutung entsprechender Leitlinien oder Leitlinienabschnitte wird damit ohne jeden Zweifel unterstrichen, die Forderung nach qualitätvollen Studien unter Alltagsbedingungen ebenso.

\section{Sieber und W. v. Renteln-Kruse}

\section{Korrespondenzadresse}

\section{Prof. Dr. C. Sieber}

Institut für Biomedizin des Alterns

Universität Erlangen-Nürnberg

Medizinische Klinik 2, Klinikum Nürnberg

Prof.-Ernst-Nathan-Str. 1, 90419 Nürnberg

sieber@klinikum-nuernberg.de

Prof. Dr. W. v. Renteln-Kruse

Zentrum für Geriatrie und Gerontologie, Universität Hamburg, Medizinisch-Geriatrische Klinik, Albertinen-Haus

Sellhopsweg 18-22, 22459 Hamburg

w.renteln-kruse@albertinen.de

\section{Literatur}

\footnotetext{
1. Aylett $\mathrm{V}$ (2010) Do geriatricians need guidelines? BMJ 341:C5340
} betagte Menschen passen aufgrund ihrer Multimorbidität nur schlecht in ein solch 


\section{Hier steht eine Anzeige.}

Springer 